\title{
Androsterone Glucuronide
}

National Cancer Institute

\section{Source}

National Cancer Institute. Androsterone Glucuronide. NCI Thesaurus. Code C120007.

A glucuronosyltransferase-modified, liver metabolite of the steroid androsterone and metabolite of the steroid dihydrotestosterone found in serum, with weak androgenic activity. 